\title{
Correction: Experimental African trypanosome infection suppresses the development of multiple myeloma in mice by inducing intrinsic apoptosis of malignant plasma cells
}

\author{
Nathan De Beule ${ }^{1, *}$, Eline Menu ${ }^{1, *}$, Mathieu J.M. Bertrand ${ }^{2,3}$, Mérédis Favreau ${ }^{1,4,9}$, \\ Elke De Bruyne ${ }^{1}$, Ken Maes ${ }^{1}$, Kim De Veirman ${ }^{1}$, Magdalena Radwanska ${ }^{3,4}$, Afshin \\ Samali ${ }^{5,6}$, Stefan Magez ${ }^{7,8}$, Karin Vanderkerken ${ }^{1}$ and Carl De Trez $^{8}$ \\ ${ }^{1}$ Department of Hematology and Immunology-Myeloma Center Brussels, Vrije Universiteit Brussel, Brussels, Belgium \\ ${ }^{2}$ Inflammation Research Center, VIB, Zwijnaarde-Ghent, Belgium \\ ${ }^{3}$ Department of Biomedical Molecular Biology, Ghent University, Zwijnaarde-Ghent, Belgium \\ ${ }^{4}$ Department of Molecular Immunology and Inflammation, VIB Inflammation Research Center, Ghent University, Ghent, \\ Belgium \\ ${ }^{5}$ Apoptosis Research Centre, NUI Galway, Ireland \\ ${ }^{6}$ School of Natural Sciences, NUI Galway, Ireland \\ 7 Ghent University Global Campus, Yeonsu-Gu, Incheon, South Korea \\ ${ }^{8}$ Department of Structural Biology Research Center (SBRC), Research Unit of Cellular and Molecular Immunology, Vrije \\ Universiteit Brussel (VUB), Flanders Institute for Biotechnology (VIB), Brussels, Belgium \\ ${ }^{9}$ Department of Internal Medicine, Ghent University, Belgium \\ * These authors have contributed equally to the work \\ Published: April 27, 2018
}

Copyright: De Beule et al. This is an open-access article distributed under the terms of the Creative Commons Attribution License 3.0 (CC BY 3.0), which permits unrestricted use, distribution, and reproduction in any medium, provided the original author and source are credited.

This article has been corrected: The correct author affiliation is given below:

\section{Mérédis Favreau ${ }^{1,4,9}$ and Magdalena Radwanska ${ }^{3,4}$}

${ }^{9}$ Department of Internal Medicine, Ghent University, Belgium

Original article: Oncotarget. 2017; 8:52016-52025. https://doi.org/10.18632/oncotarget.18152 